\title{
SIPF Protocol for Wireless Sensor Networks with Optimal Energy Efficiency Depending on Density
}

\author{
Walid BOUDHIAFI ${ }^{1 *}$ and Tahar EZZEDINE ${ }^{2}$ \\ 1,2 Sys'Com laboratory, National Engineering School of Tunis, University of Tunis - El Manar, Tunis \\ *walid.boudhiafiefsegt.utm.tn
}

\begin{abstract}
Wireless sensor networks (WSN) are viewed as a group of sensor nodes that monitor an area and interact collectively to provide data directly to the base station (BS) or intermediate sensor nodes through single-hop routing, multi-hop routing. The main characteristics of a WSN are: lifetime, rate of loss and security: The lifetime of a WSN is highly dependent on the energy consumption of the sensor nodes. This limitation makes extending the sensor node's life more difficult. In this paper, we present an upgrade to the Sink Initiated Path Formation (SIPF) protocol that minimizes power consumption and packet loss rate as a function of the density of sensor nodes in the vicinity of the "sink" sensor node.
\end{abstract}

Keywords: Duty-Cycle, Energy, Routing protocol, SIPF protocol, Wireless Sensor Networks (WSN), density.

\section{INTRODUCTION}

WSN are exploited in surveillance applications such as military, environmental, medical and commercial [1]. They consist of small nodes, deployed to collect information related to their environment. These nodes are self-contained and typically run on a battery or stack [2]. A battery cannot be recharged, because the nodes can be deployed in a hostile environment or difficult to access by humans. Indeed, energy is a very expensive and critical resource and it must be used sparingly. Therefore, a WSN must have a sufficiently long lifetime to meet the demands of the users [3].

The criticism generally levelled at these networks is the excessive power consumption which is one of the essential criteria when designing a protocol to increase the lifetime of WSN [4]. Optimizing energy consumption is difficult to achieve because it occurs at all levels: application, topology, routing. It is necessary to define relevant models to optimize energy consumption [5].

The objective of this paper is to increase the overall lifetime of WSN depending on the density of sensor nodes in the neighbours' "sink" node [6]. A "sink" node is a data collection node sent by the sensor nodes that make up WSN. In one-hop communication, if the density of sensor nodes is low around the "sink" node, these nodes go dead quickly at due to traffic, the "sink" node loses communication with the rest of the sensor nodes of the network, consequently, the network is unfunctional [7]. In this paper, we will solve this problem, using the SIPF protocol, in order to optimize the density of the sensor nodes neighboring the "sink" node.

This paper is structured as follows: In the first section, we describe the SIPF protocol [8]. The second section is devoted to the performance of the optimization of the SIPF protocol. Finally, a conclusion summarizes the present work

\section{THE SIPF PROTOCOL}

\subsection{Description}

Sink Initiated Path Formation (SIPF) is a sleep planning protocol to increase the ratio of sleep sensor nodes in the WSN [9].

The SIPF protocol is based on 2 phases: the scheduling phase and a data transfer phase. During this phase, a few nodes are powered off because their activity is unnecessary, and they are called redundant nodes.

The Self-Scheduling phase is divided into three subsections:

- First subsection: In this section: not all sensor nodes have roles, they are all regular sensor nodes. A sensor node decides to be a regular leaf sensor node or a regular central sensor node according to its coordinates, the dimension of the detection 
zone and the coordinates of the "sink" node and the scope R. Each sensor node begins by discovering its environment in order to fill its list of neighboring sensor nodes. Then, the sensor nodes move on to the second subsection [10].

- Second subsection: In this section, we start to select from the header leaf sensor node, this technique is based on the idea of selecting the neighbor furthest in the list of neighbors. In this way the number of top leaf nodes is minimized. The "sink" node selects the furthest neighbor leaf node and transmits a packet to it to inform it that it will be the next leading leaf sensor node [11]. When receiving the packet, the selected head leaf sensor node chooses the furthest neighbor as a next head leaf node among its neighbors by sending a packet and so on. These selections are made in a predefined direction as shown in Fig. 1.

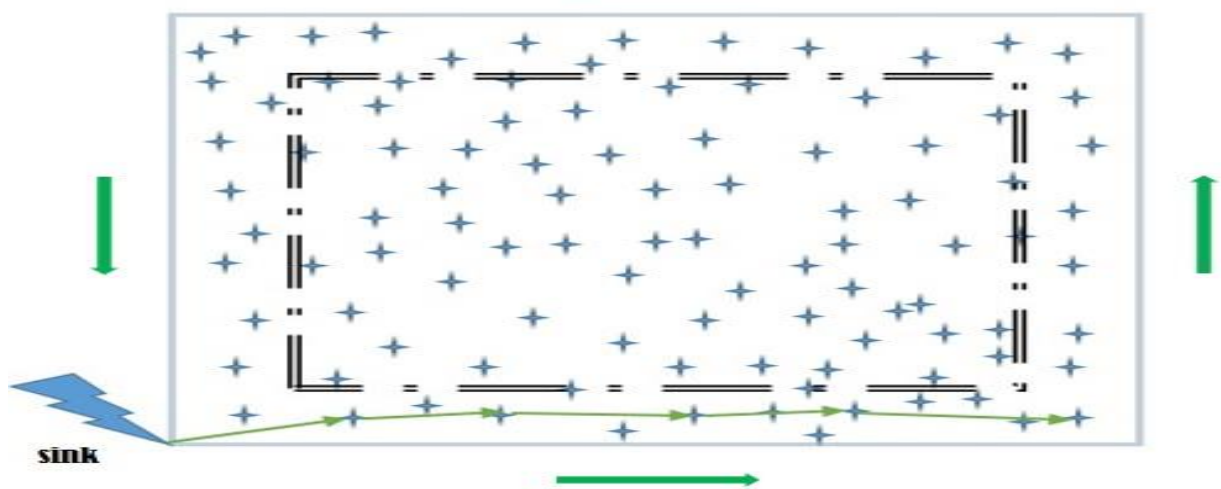

Fig.1. Selection of the head leaf sensor node

- Third subsection: The last procedure is to select the central sensor nodes. This procedure is identical to that for selecting the head leaf sensor nodes. The objective is to select the most distant neighboring sensor node directed towards the "sink". This selection is initiated by the head leaf sensor nodes located on the edge of the detection area as shown in fig. 2 .

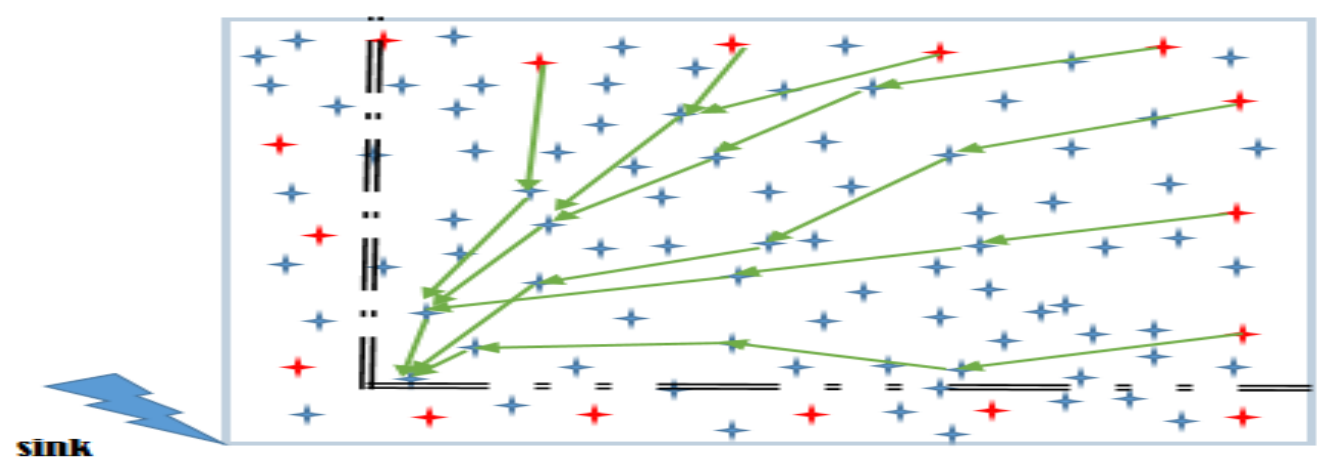

Fig.2. The selection of the central leaf node

Each node launches its selection within a period defined randomly and which varies from one node to another. Then, the node selected to be a head central node searches for its furthest neighbor in the direction of the "sink" [12]. In Fig. 3, we have schematized the algorithm of the SIPF protocol. 


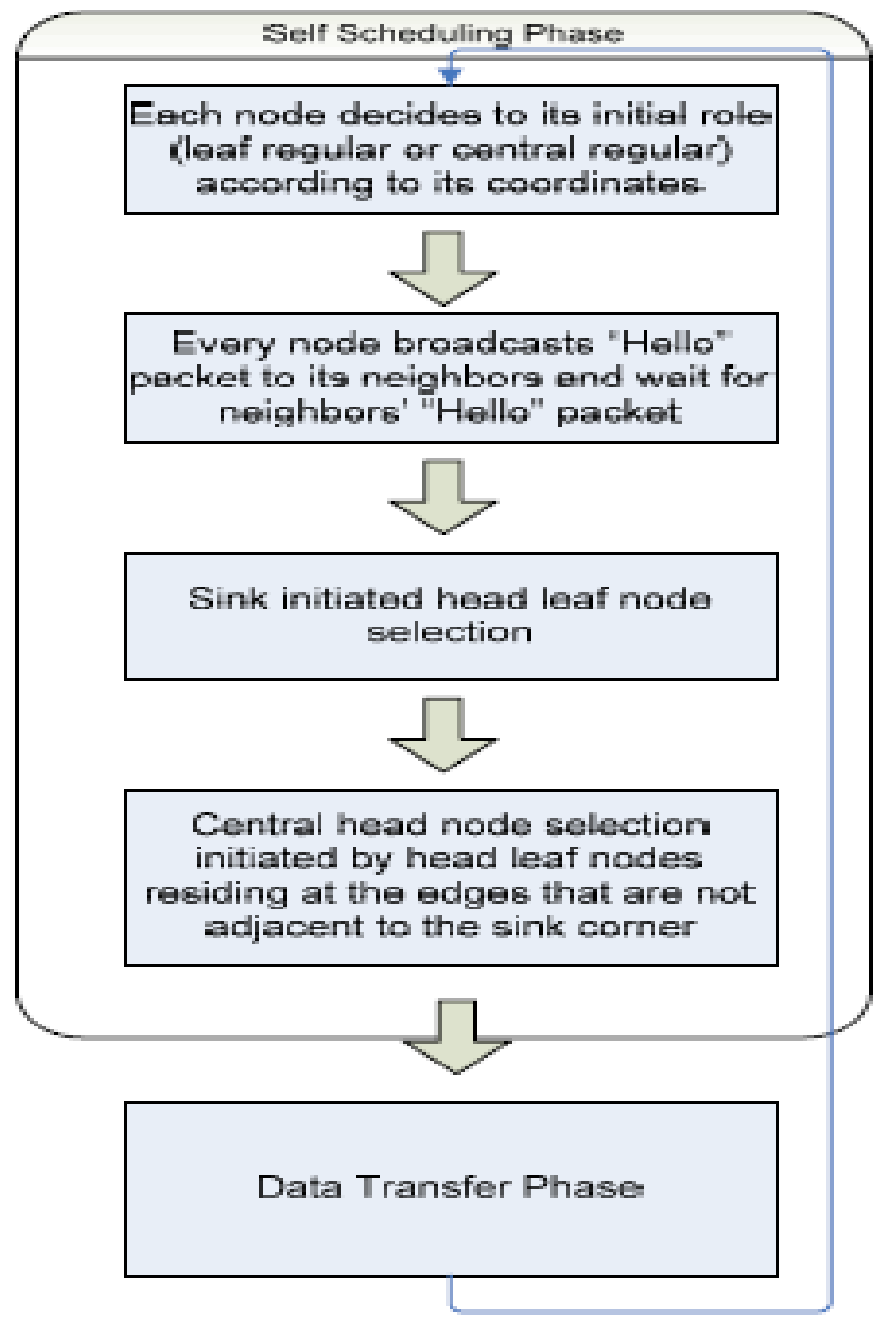

Fig. 3. SIPF Algorithm [13]

\subsection{Drawbacks}

The limits of this algorithm: if a sensor node will send information to the "sink" node and it cannot find the central sensor node or header leaf sensor node in its list of neighboring nodes in the predefined path as mentioned in the fig. 2, therefore, no sleep occurs for all sensor nodes in the network. It is for this reason that in low density networks the ratio of sleeping nodes will not be acceptable.

\section{OPTIMIZATION OF THE SIPF PROTOCOL}

In this section, we will present the simulation parameters and the ameliorations on the SIPF protocol.

\subsection{Hypothesis}

- Symmetrical communication between the sensor nodes.

- Each node knows: their geographic locations, its residual energy, its neighbors and their coordinates.

- All nodes have the same capacities.

- The deployment of sensor nodes is random.

- The failure of a node is caused only by the exhaustion of its battery.

- The lifetime of the network is presented in the form of cycles, each cycle contains two phases.

○ The first phase: this involves selecting the nodes that must remain active during a cycle.

- The second phase: during this phase we transmit the data until the death of the first node. 


\subsection{Metrics}

In table 1 presented the different parameters, we are exploited in the phase of simulation

Table 1. Simulation parameters

\begin{tabular}{|l|l|}
\hline Parameters & value \\
\hline Area $(\mathrm{m})$ & $4 * 4$ \\
\hline Radius(m) & 1 \\
\hline Size of control packet(bits) & 100 \\
\hline Size of data packet(bits) & 2000 \\
\hline E(j) & 0.5 \\
\hline Density d1 (node $\left./ \boldsymbol{m}^{2}\right)$ & 8 \\
\hline Density d2 (node $\left./ \boldsymbol{m}^{2}\right)$ & 12 \\
\hline Density d3 (node $\left./ \boldsymbol{m}^{2}\right)$ & 16 \\
\hline
\end{tabular}

\subsection{Results:}

In this section, we will study the energy consumption as a function of the number of cycles by varying the density in order to set the value which optimizes the energy consumption and gives a stable network.

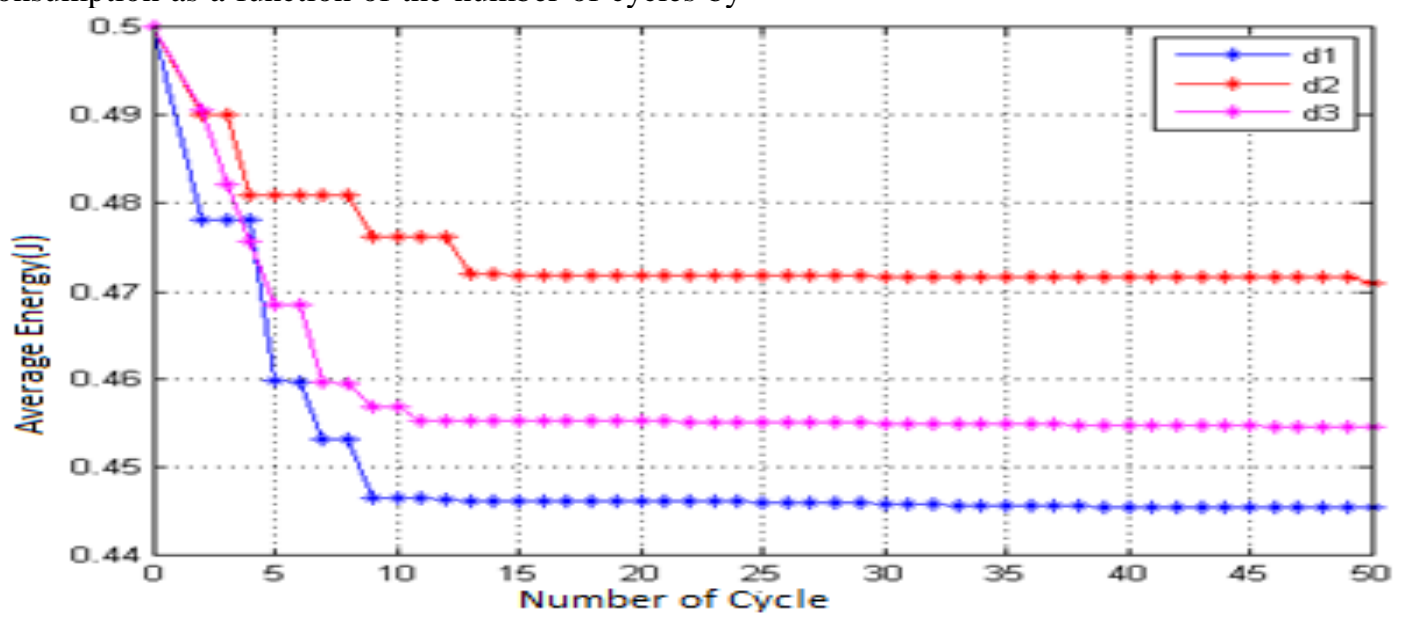

Fig.4. Average energy as a function of number of cycles with the three different densities.

Density curve d1: We have extracted the curve of the energy consumption as a function of the number of cycles: The shape of this curve shows that the energy gradually decreases and then it remains constant. But after nine cycles, the network loses connectivity which causes the network to collapse. We can deduce that after the death of eight nodes, less than $0.062 \%$ of the total number of nodes (which is determined by the quotient of the number of dead nodes over the total number of nodes in the network) is dead, the network loses connectivity. because all the exhausted nodes are neighbors of the sink. we compare the three curves; we notice that the network is exhausted very early because the neighboring nodes of the sink are all dead. But in the curve of (d1), the energy utilization is improved a little unlike the curves (d2 and $\mathrm{d} 3$ ).

From these results, we propose to fix the density at 8 nodes $/ \boldsymbol{m}^{2}$ to implement the improvement of the SIPF protocol.

\subsection{SIPF improvement}

The problem in this protocol arises during the exhaustion of all neighboring nodes at the sink. The objective of this section is to find a remedy for this problem. We have chosen to set the density at 8 nodes $/ \boldsymbol{m}^{2}$ in a detection area of $\left(4 \times 4 \boldsymbol{m}^{2}\right)$ as indicated in paper [7]. And we got the curves shown in the following figure. 


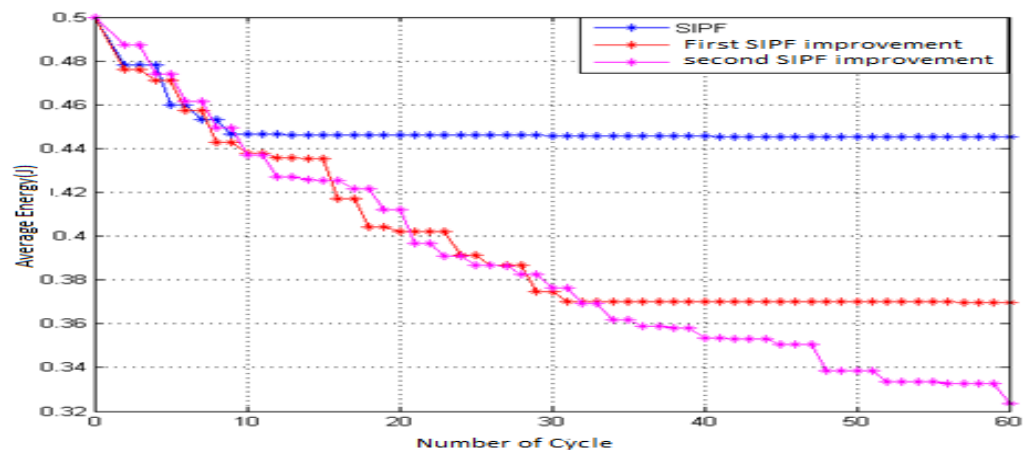

Fig.5. Comparison of the three curves (depending on the density)

- Curve of First SIPF improvement: we propose to increase the density only to the neighbors of the sink. We will have a density at the neighbors of the sink node (1):

$$
\text { Density }_{\text {sink }}=\mathrm{d}_{1}+
$$

\section{$(0,1 \times$ Total number of nodes $)$}

We notice that connectivity is lost after the death of plus or minus thirty nodes at an average energy of $\pm 0.37 \mathrm{~J}$ (instead of $\pm 0.445 \mathrm{~J}$ in Fig. 5 ). The improvement is remarkable in energy use but still insufficient as connectivity is lost without harnessing the full energy of all nodes in the network.
Curve of Second SIPF improvement: we again increase the density to 0.2 at the neighbors of the sink as described by this equation (2): Density $_{\text {sink }}=d_{1}+(0,2 \times$ Total number of nodes)

We notice an absolute improvement has occurred (relative to the curves) and connectivity is lost at sixty cycles ( 9 cycles in Fig.5).

\section{5. loss rate}

In this section, we will trace the exchange of packets after the first improvement of the SIPF protocol (density $=0.1$ in the vicinity of the "sink" node). We plotted the curve of the packet exchange and we got the following result.

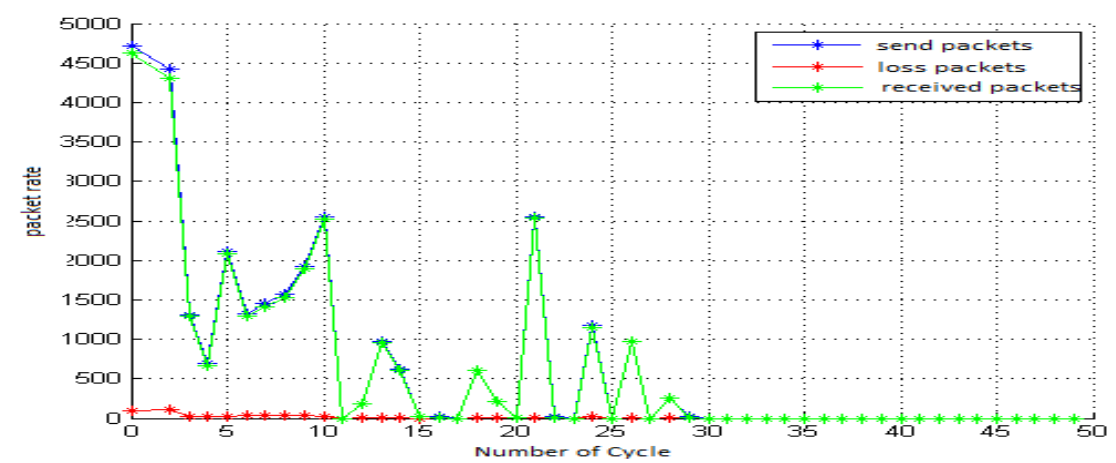

Fig.6. Packets exchanged with the first improvement of SIPF

In Fig.6, the transmission is only done if there is connectivity between the "sink" node and its neighboring nodes. The loss phenomenon is only present if there is connectivity or communication between the "sink" node and its neighboring nodes. By comparing this curve to that of the average energy (fig.5), we can conclude that the two curves are strongly linked, because from cycle number 10 the energy remains unusable and no communication between "sink" node and its nodes. neighbors

We can conclude that if the transmission rate increases, the loss rate also increases.

Finally, in fig.7, we present two comparative curves. The red curve shows the lost SIFP packets and the blue curve shows the lost SIPF packets after a 0.1 density increase in the vicinity of the sink node. 


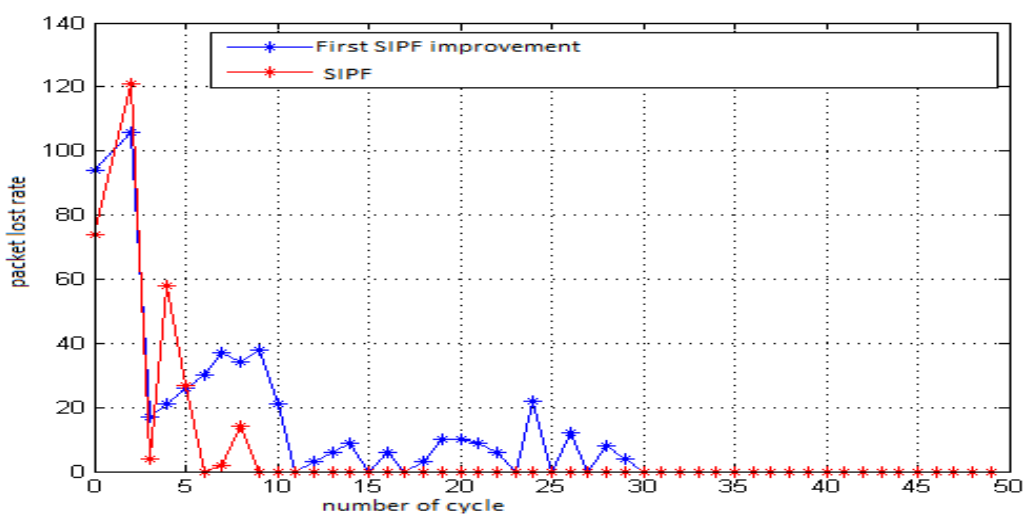

Fig.7. Comparison of losses of SIPF and First SIPF improvement

We notice that when we have packets transmitted, there is always loss and the other advantage of this protocol is that the loss rate is low.

\section{CONCLUSION}

We have presented the SIPF protocol as a protocol based on one-hop communication between sensor nodes, this communication technique shows weakness when the density of sensor nodes is low in the network, especially when the sensor nodes are not neither finds a head leaf sensor node or central sensor node in its list of neighboring nodes, therefore, no sleep occurs for all sensor nodes in the network, the nodes die quickly. We have improved the SIPF protocol to vary the density of the sensor nodes of the "sink" node as a function of the density of network sensor nodes. This idea works well when the traffic in the network is too high.

In perspective, nodes with neighbors of the "sink" node are likely to die first. For this reason, we suggest using the clustering technique or the hierarchical technique in order to exploit multi-hop routing.

\section{AUTHORS' CONTRIBUTIONS}

The basic idea in this paper is to increase the lifetime of wsn in order to improve the sipf protocol. This improvement consists in finding a density of the neighbouring nodes of the "sink" node proportional to the density of the nodes in order to increase the lifetime of wsn.

\section{REFERENCES}

[1] Walid BOUDHIAFI and $\begin{array}{r}\text { Tahar } \\ \text { EZZEDINE“Optimization of Multi-level }\end{array}$

HEED Protocol in Wireless Sensor Networks" Spring , Communications in Computer and Information Science, book series (CCIS, volume 1455), 23 October 2021, https://link.springer.com/chapter/10.1007/9783-030-89654-6_29

[2] D. Kandris, C. Nakas, D. Vomvas, and G. Koulouras, "Applications of wireless sensor networks: an up-to-date survey", Applied System Innovation,2020.

[3] K.Lin, J.Liu, Y.Zhang, P.Li "Reliable Lowpower and High Throughput Wireless Sensor Network Data Collection", IEEE wireless communications and networking 2021.

[4] R.Kaur, J.Sandhu "A Study on Security Attacks in Wireless Sensor Network", International Conference on Advance Computing and Innovative Technologies in Engineering (ICACITE),India 2021, https://ieeexplore.ieee.org/document/9404619.

[5] M. Saxena and S. Dutta, "Improved the efficiency of iot in agriculture by introduction optimum energy harvesting in wsn", in 2020 International Conference on Innovative Trends in Information Technology (ICITIIT), 2020.

[6] Y.Liu,J.Xiao, C.Li,H.Qin, and J.Zhou "Sensor Duty Cycle for Prolonging Network Lifetime Using Quantum Clone Grey Wolf Optimization Algorithm in Industrial Wireless Sensor Networks",Journal of Sensors, Volume 2021

[7] A. Hamzah, M. Shurman, O. Al-Jarrah and E. Taqieddin, "Energy-Efficient Fuzzy-Logic Based Clustering Technique for Hierarchical Routing Protocols in Wireless Sensor Networks" in Sensors (Basel) 2019.

[8] A.Ray and D.De. «Performance evaluation of tree based data aggregation for real time indoor environment monitoring using wireless sensor network." Microsystem Technologies, Springer, March 2017. 
[9] J. Parimanam,M.Rajagopal and J. Murugesan "Cluster-based Routing Protocols for Heterogeneous Wireless Sensor Networks: A Review". Computer Science and Engineering.2020

[10] M. Yaqub, S.Ahmed, S.Bouk and D. Kim "Towards energy efficient duty cycling in underwater wireless sensor networks" Spring Multimedia Tools and Applications volume 78,2019.

[11] D. Suhag, S. S. Gaur and A. K. Mohapatra, "A proposed scheme to achieve node authentication in military applications of wireless sensor network", vol. 0510, pp. 347362, 2019.
[12] A. Amer, A. Fawzy, M. Shokair, W. Saad, S. EL-Halafawy and A. Elkorany, "Proposed Sink Controlled Uniform Cluster Head Distribution Routing Protocol for WSNs" Electronic Engineering Research (MJEER), -2017.

[13] Y. Zaied;W.Saad and M.Shokair "Energy "Efficient Of Grid Clustering Based TEEN Protocol for Cognitive Radio Wireless Sensor Networks" 2021 International Conference on Electronic Engineering (ICEEM). 